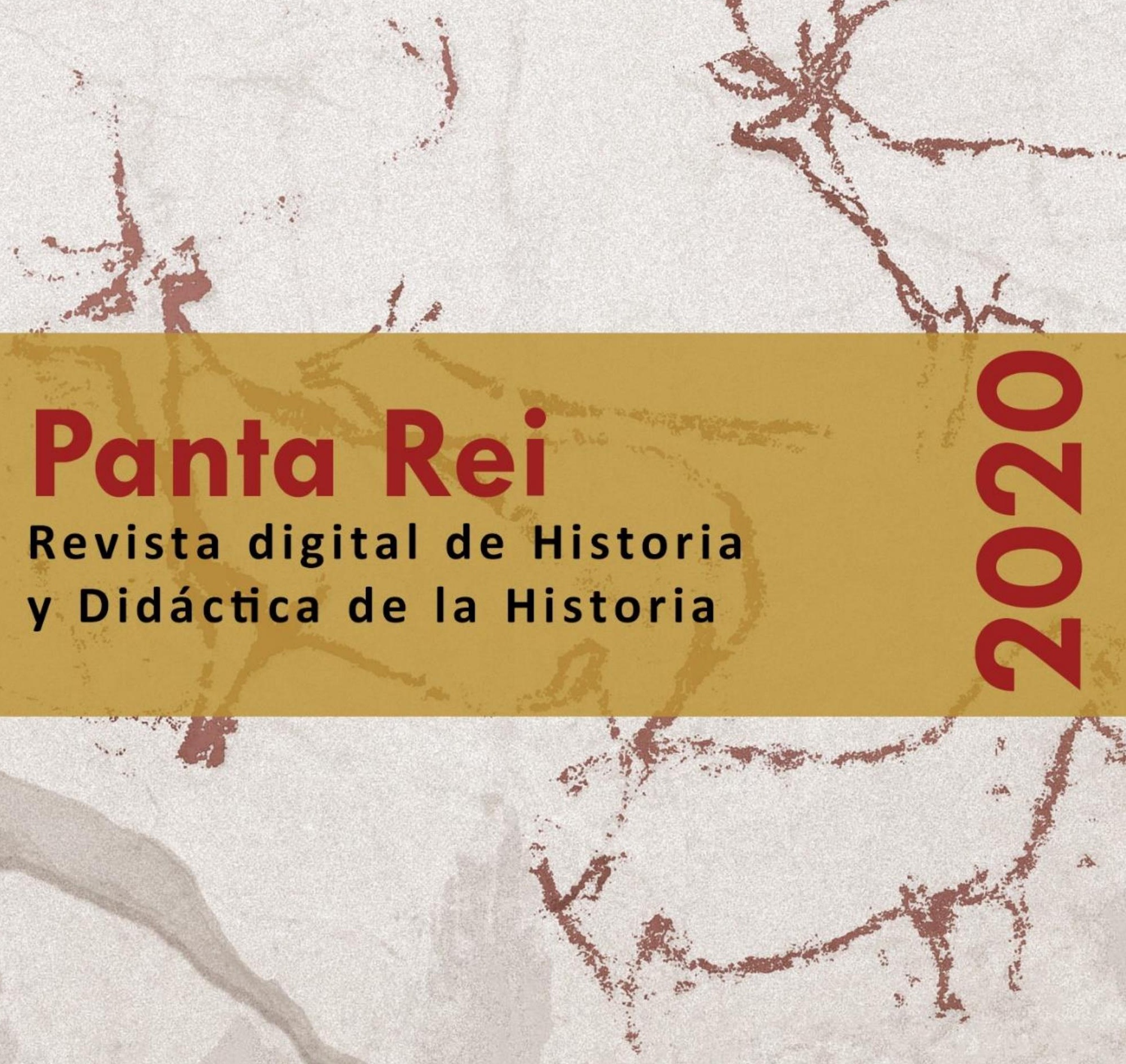




\section{0}

Revista anual

Fecha de inicio: 1995

RevistaPantaRei.pantarei@um.es

\section{Edita:}

Centro de Estudios del Próximo Oriente y la

Antigüedad Tardía - CEPOAT

Edificio Universitario Saavedra Fajardo.

Universidad de Murcia

C/ Actor Isidoro Máiquez, 9

30007 - MURCIA - ESPAÑA

Teléfono: $(+34) 868883890$

cepoat@um.es

Web: www.um.es/cepoat/pantarei

Ediciones de la Universidad de Murcia - EDITUM

Edificio Pleiades. Campus de Espinardo.

Universidad de Murcia

$\mathrm{C} /$ Campus, s/n

30100 - MURCIA - ESPAÑA

Teléfono: $(+34) 868883013$

En portada: calco de las pinturas rupestres de la editum@um.es

Web: https://www.um.es/web/editum/

Cueva del Niño. García Moreno et al., 2016.

Edición 2020

ISSNe: 2386-8864

Responsables de los textos: sus autores.

ISSN: $1136-2464$

Depósito legal: MU-966-1995

Responsable de la presente edición: Consejo Editorial de Panta Rei. 


\section{CONSEJO DE REDACCIÓN}

Coordinador editorial

Egea Vivancos, Alejandro [Didáctica de las Ciencias Sociales, Universidad de Murcia]

Secretaria

Arias Ferrer, Laura [Didáctica de las Ciencias

Sociales, Universidad de Murcia]

Editores

Jiménez Vialás, Helena [Historia Antigua, Universidad de Murcia]

Martínez Gil, Tània [Didáctica de las Ciencias

Sociales, Universidad de Barcelona]

Meseguer Gil, Antonio José [Historiador, Profesor de

Secundaria]

Ortiz García, Jónatan [Arqueología, Universidad de

Alcalá de Henares]

Romero Molero, Alberto [Arqueología, Universidad

Isabel I]

Sáez Giménez, David Omar [Historiador, Profesor

de Secundaria]

Sáez Rosenkranz, Isidora V. [Didáctica de las

Ciencias Sociales, Universidad de Barcelona]

Sánchez Mondejar, Celso Miguel [Arqueólogo,

Patrimonio Inteligente]

Responsable informático

Martínez García, José Javier [CEPOAT, Universidad de Murcia]

Responsables de traducción y corrección lingüística Martínez Martínez, Cristina [Profesora de Secundaria, Sociedad Española de Lenguas Modernas] Albaladejo Albaladejo, Sara [ISEN-Universidad de Murcia]

\section{CONSEJO ASESOR}

Adroher Auroux, Andrés María [Arqueología, Universidad de Granada]

Albero Muñoz, $M^{a}$ del Mar [H. ${ }^{a}$ del Arte, Universidad de Murcia]

Alia Miranda, Francisco [Historia Contemporánea, UCLM]

Arciniega García, Luis [Historia del Arte, Universidad de Valencia]

Barrio Barrio, Juan Antonio [Historia Medieval,

Universidad de Alicante]

Castellano i Solé, Núria [Egiptología, Schola

Didàctica Activa S.L.]

Chapman, Arthur [History Education, University

College of London, Reino Unido]

Cid López, Rosa María [Historia Antigua, Universidad de Oviedo]

Cobacho López, Ángel [Derecho, Universidad de Murcia]

Cuenca López, José María [Didáctica de las Ciencias Sociales, Universidad de Huelva]
Egea Bruno, Pedro M. ${ }^{a}$ [Historia Contemporánea, Universidad de Murcia]

Feijoo Martínez, Santiago [Arqueología, Consorcio

Ciudad Monumental de Mérida]

García Atienzar, Gabriel [Prehistoria, Universidad de

Alicante]

Ginestí Rosell, Anna [Filología Clásica, Katholische

Universität Eichstätt-Ingolstadt]

González Monfort, Neus [Didáctica de las

Ciencias Sociales, Universidad Autónoma de

Barcelona]

González Soutelo, Silvia [Arqueología, Universidad de Vigo]

Haber Uriarte, María [Prehistoria, Universidad de Murcia]

Hernández de la Fuente, David [Filología Clásica, Universidad Complutense]

Hutson, Scott R. [Anthropology, University of Kentucky, EEUU]

Igual Luis, David [Historia Medieval, UCLM]

Irigoyen López, Antonio [Historia Moderna,

Universidad de Murcia]

Jover Maestre, Francisco Javier [Prehistoria,

Universidad de Alicante]

Mahony, Simon [Digital Humanities, University College of London, Reino Unido]

Marsilla de Pascual, Francisco Reyes [Técnicas

historiográficas, Universidad de Murcia]

Martínez-Burgos García, Palma [H. ${ }^{a}$ del Arte, UCLM]

Mathis, Christian [Didaktik der Geschichte, PH Zürich]

Miralles Maldonado, José Carlos [Filología Clásica,

Universidad de Murcia]

Molina Gómez, José Antonio [Historia Antigua,

Universidad de Murcia]

Mónica Ghirardi [Historia Moderna, Universidad

Nacional de Córdoba, Argentina]

Navarro Espinach, Germán [Historia Medieval,

Universidad de Zaragoza]

Noguera Celdrán, José Miguel [Arqueología,

Universidad de Murcia]

Ortiz Heras, Manuel [Historia Contemporánea, UCLM]

Panzram, Sabine [Historia Antigua, Universität

Hamburg]

Pérez Molina, Miguel Emilio [Filología Clásica,

Universidad de Murcia]

Prados Martínez, Fernando [Arqueología,

Universidad de Alicante]

Sánchez lbáñez, Raquel [Didáctica de las Ciencias

Sociales, Universidad de Murcia]

Sancho Gómez, Miguel Pablo [Educación, UCAM]

Victoria Moreno, Diego [Historia Contemporánea,

UNED]

Vilar García, María José [Historia Contemporánea,

Universidad de Murcia]

Vivas Sainz, Inmaculada [H. ${ }^{a}$ del Arte, UNED]

Zamora López, José Ángel [Próximo Oriente Antiguo,

CCHS-CSIC] 



\section{Índice}

\section{Artículos}

Las ocupaciones paleolíticas en el sur de la provincia de Albacete

Noelia Sánchez Martínez

El elefante en las acuñaciones hispanocartaginesas

José Luis Aledo Martínez

La epigrafía votiva romana de Caldas de Montbui (Vallés Oriental, Barcelona) (ss. I-II d. C.). Un ejemplo de promoción de las élites provinciales de la tarraconensis en centros de aguas mineromedicinales

Jesús Sánchez Alguacil

Representación de la historia de España por medio de la filatelia. Estudio de los sellos diseñados por Gallego y Rey

Pedro Vázquez-Miraz

La representación del patrimonio arqueológico en los libros de texto de Educación Primaria: EI 109 contexto indígena canario como estudio de caso

A. José Farrujía, Carmen Ascanio Sánchez, Ulises Martín Herández y Cristo Manuel Hernández Gómez

La empatía como elemento para la adquisición del pensamiento histórico en alumnos de 129 bachillerato. Un estudio de caso centrado en la Guerra Civil española y el franquismo Sebastián Molina Puche y Adrián Salmerón Ayala

Fuentes orales para el desarrollo de la empatía histórica: un estudio en la formación del 155 profesorado de Educación Infantil

$M^{a}$. Teresa Carril-Merino, Beatriz Andreu-Mediero, Mercedes de la Calle Carrecedo y Esther López Torres

¿Qué aporta el género a la formación de docentes de historia? La valoración de la perspectiva de género entre los estudiantes del Máster de Profesor/Profesora en Educación Secundaria Helena Rausell Guillot

Educar para una ciudadanía crítica: una investigación a partir de los usos y finalidades de la historia escolar

Néstor Banderas Navarro

\section{Reseñas}

A. Brilli (2018). El viaje a Oriente, Madrid: A. Machado Libros, 390 págs Juan Álvarez García

Altamira (Hugh Hudson, 2016) 



\title{
A. Brilli (2018). El viaje a Oriente, Madrid: A. Machado Libros, 390 págs. ISBN: 9788477748182
}

\author{
[Traducción de Juan Antonio Méndez del original publicado en 2009, II \\ viaggio in Oriente, Bologna: Societò editrice il Mulino]
}

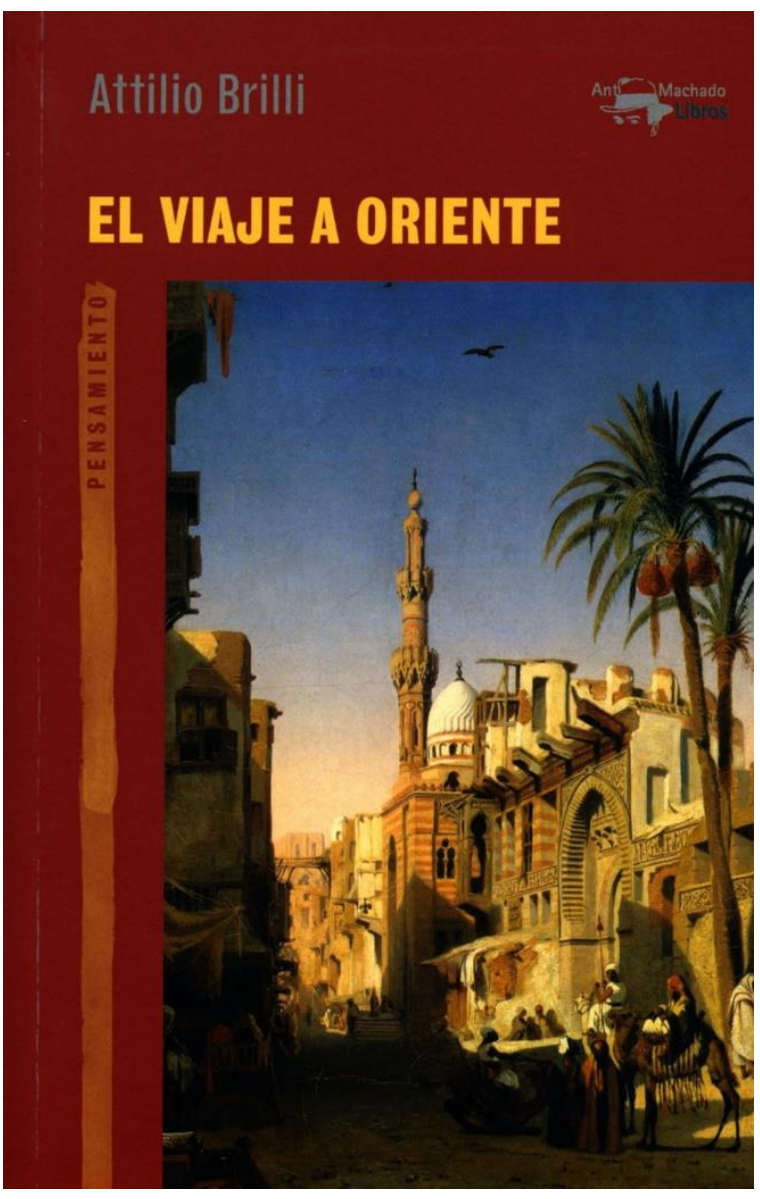

Fuente: Editorial Antonio Machado.
El viaje, entendido como exploración y búsqueda de lo desconocido, puede constituir una de las más apasionantes actividades del historiador. Si contemplamos el trabajo historiográfico desde esta perspectiva, podemos observar los grandes viajes que desarrollaron historiadores cuya obra se encuentra entre las más destacadas de nuestra disciplina. Sin ir más lejos, todavía nos maravillamos con la prosa de Heródoto, quien en sus viajes investigaría y narraría la historia de civilizaciones tan antiguas como la egipcia y ciudades tan grandiosas como Babilonia. Tras él siguieron los pasos de militares romanos que dejaron constancia de sus hazañas y de los pueblos a los que combatieron. Tampoco podemos olvidar las sendas mil veces andadas de los peregrinos medievales de diferentes confesiones en sus travesías hacia las ciudades santas, así como las de los comerciantes y exploradores en busca de nuevos lugares

donde hacer negocio, desde Benjamín de Tudela a Marco Polo. A su vez, con la llegada de la revolución geográfica y la ampliación de los horizontes, llegaron los exploradores de época moderna y las descripciones de sus viajes por nuevos continentes; para finalizar en sus últimos herederos, las misiones científicas de los siglos XIX y XX encomendadas por las grandes potencias europeas. Así pues, podemos considerar el viaje como una labor de campo fundamental para el historiador $y$, las narraciones de los mismos, una fuente histórica de gran valor. De tal manera que incluso el propio viaje debe ser considerado objeto de reflexión e indagación histórica, una indagación en la que destaca la obra de Attilio Brilli. 
Considerado uno de los mayores expertos en literatura de viajes, Attilio Brilli es profesor de literatura inglesa en la Universidad de Siena. De entre sus mayores éxitos literarios se encuentran Quando viaggaiare era un'arte (1995), Il viaggiatorie immaginario (1997) o el reconocido II viaggio en Italia, Storia di una grande tradizione culturale (2006), galardonado con los prestigiosos premios Hemingway de 2006 y Lawrence de 2007.

Esta vez, el autor nos sumerge en una de las grandes epopeyas de la tradición europea, el viaje al Oriente, un Oriente al que se consideraba un territorio inexplorado que debía ser descubierto o redescubierto, ya fuera a través de expediciones científicas, misiones de carácter diplomático o comercial o aventuras personales. El autor plantea una serie de interrogantes en la introducción a la obra que irá desglosando y respondiendo a lo largo de los ocho capítulos en los que organiza el libro: ¿̇cuál es el significado del viaje al Oriente?, ¿qué representa la literatura orientalista?, ¿qué buscaba el viajero al Oriente y qué provoca el Oriente en el viajero?, ifue únicamente una búsqueda de lo desconocido o consiguió generar en el viajero una serie de replanteamientos de los propios esquemas occidentales? y, finalmente, żexistió ese oriente descrito? Para contestar a estas preguntas, la materia prima sobre la que va a trabajar el autor no es tanto la literatura de viajes sino el conjunto de notas, informes, diarios y cartas escritas por los viajeros. Se trata de una perspectiva interesante al ser una información primaria $y$, en palabras del autor, "sin filtrar" en gabinetes y despachos.

Podemos agrupar los ocho capítulos de este libro en tres partes. Una primera parte que reuniría los dos primeros capítulos en los que el autor presenta a sus protagonistas: por un lado, el propio Oriente descrito en la literatura de viajes y, por otro, aquellos viajeros que le dieron forma. Una segunda parte englobaría los capítulos tres, cuatro y cinco en donde el autor desarrolla las líneas maestras de ese "viaje a Oriente": los temas sobre los que se trabaja en la literatura de viajes al Oriente, las producciones culturales y artísticas del mismo y en qué consistía el propio viaje. Finalmente, la tercera parte recogería los capítulos seis, siete y ocho y en ellos se describen los viajes y experiencias de ciertos viajeros que, por su fama y por las implicaciones que tuvo su trabajo, el autor ha considerado importante analizar en profundidad.

El primer capítulo, comienza con la propia literatura de viajes, que no es sino la portadora de la imagen de Oriente que se difundió por Europa durante los siglos XVIII, XIX y XX. Posiblemente, la principal influencia de esta literatura se deba a la traducción y difusión de Las mil y una noches, obra que será elevada a la categoría de manual de referencia para comprender dicho Oriente. La traducción de esta obra literaria por parte de A. Galland a comienzos del s. XVIII se realizó sobre manuscritos y recopilaciones de época turca, los cuales incluyeron en Las mil y una noches originales obras que, en su momento, debieron ser cuentos independientes como las aventuras de Simbad el marino, Aladino y la lámpara maravillosa o Alí Baba y los cuarenta ladrones; por lo que podemos pensar que posiblemente el compendio de relatos se adaptó a los gustos del Serrallo y se incluyeron imágenes y estilos propios de época otomana. Para un estudio de esta obra recomendamos la introducción a la edición de R. R. Khawan de 1986 y traducida al español en 2010 (Las mil y una noches, Barcelona: Edhasa). 
Así pues, la literatura orientalista se define por contar con un alto componente sensual, destacando los olores, los colores, los sabores y las formas. Por otro lado, estableció una neta distinción entre el Oriente atrasado, mítico y fabuloso frente a la Europa moderna y al frente de las innovaciones políticas, sociales, económicas y tecnológicas. Estas obras darán la imagen del Oriente como un todo homogéneo y dotado, en cierta medida, de coherencia política por la dominación otomana, cuyas instituciones políticas, así como sus prácticas sociales y culturales, serán descritas por los viajeros como totalmente opuestas a los valores occidentales. El autor finaliza este capítulo señalando una serie de lugares emblemáticos que se convierten en recurrentes en esta literatura: los puertos europeos del Mediterráneo y Grecia como salto previo al Oriente, Constantinopla/Estambul como su acceso principal, ciudades como Beirut, Damasco, Alejandría, El Cairo y la rivera del Nilo, así como Jerusalén para aquellos viajeros interesados en visitar los Santos Lugares, Medina y la Meca para los más arriesgados y las antiguas ciudades de las civilizaciones que hollaron esta tierra en Siria y Mesopotamia, destacando las descripciones de las ruinas de Palmira.

Por su parte, el capítulo dos se centra en los viajes y obras de aquellos pioneros que, desde finales del s. XVII y durante los siglos XVIII, XIX y XX, encontraron en el Oriente la fuente de inspiración para sus libros de viajes. Posiblemente podamos situar el momento clave en el que esta tradición se generaliza en Europa en las campañas napoleónicas en Egipto (1798-1801), a la cual también asistieron científicos y eruditos que acabarían componiendo aquella Description de l'Egypte. El autor se centra seguidamente en aquellos que luego vendrían a colonizar estas mismas tierras tras la desaparición del Imperio Otomano, ingleses y franceses; sin dejar de lado a viajeros italianos y norteamericanos.

El capítulo tres está dedicado a los temas recurrentes que inundan la literatura orientalista. Entre ellos se encuentra el tema del despotismo oriental manifestado no solo en la figura del sultán, definido como señor omnipotente y arbitrario que gobierna su imperio desde Estambul, sino también a través de la jerarquía piramidal a base de funcionarios y militares que, desde el Serrallo a las provincias, controlan igualmente el estado. En este sentido, la literatura orientalista suele describir con gran detalle los abusos por parte de este estado, al que considera parasitario de la sociedad que gobierna e incapaz de redistribuir recursos o servicios. Otro de los temas es el harén del sultán, un espacio hipnótico vedado a los hombres quienes dejan volar su imaginación en descripciones de gran sensualidad, resaltando los olores, los colores y las formas que invaden los sentidos del viajero. Como extensión de este último, también se destaca en la literatura orientalista las imágenes del baño turco. Igualmente, son de gran interés para el viajero los ropajes que vienen a identificar a las gentes y culturas que el Imperio Otomano llegó a gobernar, destacando las vestimentas femeninas. Pero también muchos describirán otras gentes que quedaron al margen de la vida y el ajetreo urbanos, como los beduinos del desierto, cuya imagen y vida desarraigada atrapa especialmente el corazón romántico de los viajeros europeos. En definitiva, podemos decir que los temas que más destacaron los viajeros al Oriente fueron aquellos tan singularmente diferentes del occidente europeo que se transformaron en estereotipo inmóvil y definitorio de toda esta región.

En el capítulo cuarto, el autor dedica unas páginas a otra de las grandes manifestaciones artísticas, la pintura orientalista. Los pintores orientalistas recurrieron, al igual que los 
literatos, a descripciones costumbristas de la vida del Oriente, recreando típicas escenas que ellos mismos habían percibido y que así lo transmitieron en sus notas, diarios y cartas. Pero también recurren a paisajes y ruinas que así mismo generaron una profunda impresión en ellos. De esta influencia tampoco estuvo exenta la fotografía, en la que también se recrearon estas escenas.

El capítulo cinco se centra en el viaje en sí. Nos referimos a las descripciones de los caravasares en donde los viajeros se hospedan, los traductores que les guían (destacando entre ellos la figura del dragomán), las descripciones de caravanas comerciales y de peregrinos a las que se suman estos viajeros, así como del equipo con el que viajan, la escolta que los acompaña y las cabalgaduras que montan, de entre las que destaca el dromedario, símbolo del viaje a Oriente y que ha pasado al imaginario colectivo como la instantánea que se nos viene a la mente al oír esa palabra. Igualmente, no faltan las descripciones de los peligros que atenazan al viajero, desde enfermedades hasta los asaltos de bandidos. El viaje por el desierto y su inmensidad es descrito con gran detalle, aunque también son recurrentes las descripciones de los desplazamientos por mar. El autor cierra este capítulo con la mecanización de estas travesías y la llegada del ferrocarril y el vapor, a lo que se sumó la creación de las primeras empresas turísticas que, al permitir un viaje sin peligros y verdaderas aventuras, dieron por finalizada toda una tradición intelectual y artística.

Finalmente, como hemos señalado previamente, en los tres últimos capítulos de la obra, el autor analiza con mayor profundidad la experiencia de varios viajeros al Oriente que él considera destacados y en cuyos relatos se ponen de manifiesto todos los ingredientes que se han descripto previamente. Así pues, en el capítulo seis el autor describe los viajes a Egipto de J. L. Buckhardt y su estancia en El Cairo, el del pintor orientalista C. Gleyre en busca de paisajes y escenas orientalistas, el de R. Curzon en busca de códices en monasterios coptos, o los desvaríos amorosos de G. de Nerval en Egipto y Siria.

Por su parte, el capítulo siete se concentra en viajes y aventuras a la región que ya fue considerada como la más inhóspita, la península de Arabia. Comienza con la descripción de la expedición científica danesa relatada por $C$. Niebuhr quien, junto a otros eruditos ilustrados, recorrió Arabia y Yemen a mediados del siglo XVIII. De su relato destacan las fatalidades de este viaje del que únicamente sobrevivió su narrador. El capítulo continúa con la arriesgada exploración por parte de R. Burton a las ciudades santas del islam en 1863, Medina y La Meca, prohibidas a los no creyentes. Este explorador narra cómo se disfraza de musulmán para poder acceder a estas ciudades mezclado entre sus peregrinos y así poder hacer una detallada descripción de sus calles y monumentos. El capítulo finaliza con los viajes de C. Guarmani, por un lado, y el de Lady Blunt, por otro, al Neged en busca de caballos árabes que, desde mediados del s. XIX, eran altamente cotizados en Europa. Ambos, en diferentes momentos y procedentes de distintos países, recogieron una prolífica descripción de las tribus beduinas del norte de Arabia. Hay que señalar también que, en este capítulo, el autor nos regala una sucinta pero esclarecedora definición de orientalismo: "el conjunto de las representaciones de Oriente elaboradas por los países occidentales". 
Por último, esta parte, y el libro en sí, finaliza con los viajes y experiencias de aquellos viajeros que posiblemente dejaron más de sí mismos en su obra tras imprimir el Oriente en ellos una profunda huella, es el caso del viajero ilustrado C.-F. de Volney, el literato F.-R. Chateaubriand o el diplomático A. de Lamartine, cuya sensibilidad religiosa impregna las reflexiones y descripciones de su viaje.

Esta obra en su conjunto constituye un paso más en la deconstrucción del mito oriental. Este mito generado desde los países occidentales, que comprendía las regiones y culturas del Oriente como un todo homogéneo y estático, no fue sino la creación de una identidad para aquello considerado completamente ajeno $y$, desde la perspectiva eurocéntrica, inferior. Así pues, el autor, al describir objetivamente y en profundidad los diferentes aspectos que constituyeron esta tendencia literaria y artística desde los documentos y pruebas más primarios (notas, cartas, diarios, etc.), muestra la naturaleza construida y estereotipada la literatura orientalista. Los autores orientalistas recurrieron a escenas representativas y sensuales para definir ese Oriente $y$, al distribuir sus obras por los círculos eruditos de Europa, generaron una imagen radicalmente opuesta al occidente europeo. En relación a esta cuestión, destacamos el trabajo de E. Said, Orientalismo (1997), que constituye una obra de referencia en lo que respecta a la creación del mito oriental. En esta obra el pensador poscolonial ya reflexiona sobre la heterogeneidad y dinamismo de las sociedades que componen la región del Próximo Oriente y el norte de África frente a la visión inmóvil y homogénea que describe la literatura orientalista.

Por otra parte, la obra de A. Brilli también es un trabajo introductorio a diferentes disciplinas como la asiriología o la arqueología del Próximo Oriente antiguo. En este sentido, ofrece una contextualización para comprender el nacimiento de estas ciencias, puesto que muchos de los primeros viajeros al Oriente, ya fueran aventureros, diplomáticos o comerciantes, dejaron constancia de ruinas y antiguas ciudades en sus anotaciones y diarios. De hecho, el propio autor destaca, en el capítulo dos, los trabajos de A. H. Layard en las antiguas ciudades asirias, posiblemente por el hecho de que sus publicaciones fueran mucho mejor recibidas por el público europeo de la época al tratarse de ediciones de carácter mucho más comercial que aquellas de otros pioneros de la arqueología del Oriente Próximo como P. E. Botta o V. Place, las cuales, por el contrario, gozaban de una mayor calidad científica en la comprensión del espacio y el trabajo arqueológico. No obstante, dentro de esta literatura de viajes, pero alejada de lo que el propio autor denomina "locura orientalista", debemos mencionar la aventura alemana en Oriente, cuyas aportaciones al campo de la arqueología y la arquitectura oriental están igualmente salpicadas de impresiones y reflexiones sobre las regiones en las que trabajaban y las gentes que las poblaban, tal y como podemos apreciar en la obra de W. Andrae, Memorias de un Arqueólogo (1961).

Es posible que en este libro se echen en falta algunas páginas acerca de un Oriente no tan próximo, pero en torno al cual igualmente voló la imaginación de viajeros y aventureros. Nos referimos a las tierras del sur de Iraq y de Persia. La primera es recurrentemente mencionada por viajeros y exploradores, dejando constancia de la decadencia en la que se había sumido la otrora gloriosa ciudad de Bagdad en la que se ambientan tantas historias de Las mil y una noches. Por su parte, Persia evocaba en los eruditos europeos la idea de un territorio igualmente exótico y desconocido. En este 
sentido podríamos citar los viajes del diplomático A. de Rivadeneyra quien, con gran precisión y maestría literaria, deja constancia de sus viajes por Irán en dos sendas obras: Viaje de Ceilán a Damasco (1871) y Viaje al interior de Persia (1880). Así como también las expediciones científicas de $\mathrm{H}$. Rawlinson a la Roca de Behistun, cuyos trabajos en esta inscripción darían como resultado el desciframiento del cuneiforme. Del mismo modo, aunque invadida de ese espíritu orientalista, J. Dieulafoy publicaría su novela Parysatis (1890) tras su viaje, exploración y trabajos arqueológicos en el Gran Tell de Susa en el suroeste de Irán, inspirada por los restos del palacio persa que, junto a su marido M.-A. Dieulafoy, allí descubrieron.

Ciertamente, los viajes y viajeros por estas regiones del oriente no tan próximo podrían dar lugar a otra publicación como la que A. Brilli aquí nos propone. Por lo tanto, debemos considerar El Viaje a Oriente como una obra de gran precisión histórica por el volumen de documentación manejado por el autor y por el estilo claro y conciso con el que aborda una de las grandes manifestaciones artísticas, literarias e intelectuales del occidente europeo.

Juan Álvarez García Universidad Autónoma de Madrid juan.alvarez@uam.es (i) 0000-0002-4106-6044 


\section{Panta Rei}

PANTA REl es una revista digital de investigación orientada a la Historia y la Didáctica de la Historia. Su principal objetivo es la transmisión del conocimiento científico, dando una oportunidad también a los jóvenes investigadores que quieren abrirse camino en el estudio de las ciencias humanas y sociales. Se compone de estudios originales relacionados con la disciplina histórica así como su didáctica y difusión. Las diferentes secciones que componen la revista son: artículos de investigación, entrevistas a profesionales, recensiones de monografías de actualidad y crónicas de congresos o eventos científicos relevantes.

Todos los artículos publicados son objeto de un proceso de revisión a cargo de un mínimo de dos evaluadores, que se consideran expertos en el ámbito temático del artículo propuesto. Nuestro deseo es poder ofrecer unos contenidos rigurosos, de calidad y de interés.

El CEPOAT (Centro de Estudios del Próximo Oriente y la Antigüedad Tardía de la Universidad de Murcia) es la institución encargada de la coordinación y gestión de la revista, desde donde anualmente se lanzará la convocatoria para aquellos que estén interesados en publicar sus trabajos, siempre relacionados con la Historia y la Didáctica de la Historia.

PANTA REI is a digital journal focused on History and Teaching History. Its main objective is the transmission of scientific knowledge by giving also an opportunity to young researchers who want to make their way in the study of human and social sciences. It is composed by original studies related to History, as well as its didactics and promotion. The different sections of this journal are: research articles, interviews to professionals, recensions on monographs about current issues and reports about congresses or relevant scientific events.

All the articles published are subject to a revision process carried out by a minimum of two reviewers who are considered to be experts in the field of the article proposed. Our wish is to offer rigorous contents with quality and being of interest to the reader.

CEPOAT (Centre of Studies of the Middle East and Late Antiquity of the University of Murcia) is the institution in charge of the coordination and management of this journal. This is the centre from where the call for papers will be launched annually for all the people interested in publishing their papers, always related to History and Teaching History. 


\section{Normas de publicación}

El autor se compromete a enviar trabajos originales, que no se encuentren publicados en otras revistas ni en otros idiomas. Así mismo, el mismo artículo no podrá ser presentado en otras revistas mientras dure el proceso de evaluación.

\section{Envío y presentación de originales}

Las normas de edición y forma de envío de artículos a la revista se pueden consultar en https://revistas.um.es/pantarei/

Para la redacción de los trabajos se tendrá en cuenta el Manual de la American Psychological Association, en su $7 .^{a}$ edición. La extensión máxima de los trabajos será de 25 páginas. La revista acepta originales escritos en español o inglés.

\section{Proceso de valoración y evaluación}

Una vez recibidos los trabajos, la Revista realizará una primera valoración. Si el trabajo enviado se ajusta a las normas de presentación propuestas, la temática es coincidente con la línea editorial de la revista y posee la calidad científica necesaria, será remitido al consejo asesor para una primera evaluación. Si no es así en este primer paso se puede rechazar directamente los documentos que incumplan claramente la línea editorial.

Será el Consejo Asesor quien indique a la revista la originalidad, relevancia, estructura, redacción, aparato bibliográfico, etc. del trabajo enviado y, para ello, se designará a dos revisores expertos externos que evaluarán cada uno de los trabajos, que pueden formar parte (o no) de este Consejo Asesor. La selección de los revisores se ajustará a la temática y características metodológicas del trabajo. El nombre y filiación de los autores serán eliminados del trabajo para su revisión, así como los revisores actuarán de manera anónima y confidencial.

Los revisores deberán rellenar un informe de evaluación que centrará su atención en aspectos tales como características formales, originalidad y novedad de los trabajos, relevancia de las propuestas y los resultados, calidad metodológica y validez científica.

Una vez terminado el proceso se decidirá la aceptación o no de los mismos y su publicación en el número que sea pertinente, así como las modificaciones susceptibles de ser realizadas para su final publicación. Dicha notificación se enviará únicamente por correo electrónico, en un plazo máximo de seis meses. 


\section{Publishing rules}

The author is committed to submit original papers not having been published in other reviews or in other languages. In this way, it is not allowed for the same paper to be presented in other reviews during the evaluation process.

\section{Submission and presentation of originals}

The editing rules and the guidelines for the submission of papers can be consulted at https://revistas.um.es/pantarei/

The seventh edition of the Manual of the American Psychological Association will be taken into account for the writing of the papers. The length of the submitted papers will not exceed the 25 pages. The journal accepts originals written in Spanish or English.

\section{Examination and assessment process}

The Journal will submit the papers to a first examination once received. If the paper follows the presentation guidelines, the subject agrees with the editorial line of this journal, and possess the scientific quality required, it will be sent to the advisory council for a first assessment. If not, the documents which clearly fail to complete the editorial line may be rejected straightaway in this first step.

The Advisory Council will indicate the originality, relevance, structure, writing, bibliography, etc. of the text to the journal; for this purpose, two outside experts will be designated to review the papers; these experts can be (or not) part of this Advisory Council. The selection of the experts will adjust to the subject and methodological characteristics of the paper. Name and affiliation of the author will be eliminated from the text for its review, in this way experts will act anonymously and confidentially.

The experts will fill out an assessment report which will focus on aspects such as formal characteristics, originality and novelty of the papers, relevance and results of the proposal, methodological quality and scientific validity.

Once the process is finished, the acceptance or not of the papers and its publication in the corresponding edition will be decided, as well as the modifications that may be done for its final publication. This notification will be sent by email within 6 months maximum. 


\section{cepoAt edit.um}

